\title{
Selection of Elephant-Grass Genotypes for Forage Production
}

\author{
Larissa S. A. Schneider ${ }^{1}$, Rogério F. Daher ${ }^{1}$, Bruna R. S. Menezes ${ }^{2}$, Rafael S. Freitas ${ }^{1}$, Liliane B. Sousa ${ }^{1}$, \\ Verônica B. Silva ${ }^{1}$, Eduardo P. Furlani ${ }^{3} \&$ Ana K. F. Vidal ${ }^{1}$ \\ ${ }^{1}$ Universidade Estadual do Norte Fluminense Darcy Ribeiro, Campos dos Goytacazes, RJ, Brazil \\ ${ }^{2}$ Universidade Federal Rural do Rio de Janeiro, Seropédica, RJ, Brazil \\ ${ }^{3}$ Universidade Federal de Juiz de Fora, Juiz de Fora, RJ, Brazil \\ Correspondence: Bruna R. S. Menezes, Universidade Federal Rural do Rio de Janeiro, Seropédica, RJ, Brazil. \\ Tel: 55-2198-7933-632.E-mail: brunamenezes@ufrrj.br
}

$\begin{array}{lc}\text { Received: May 30, 2018 } & \text { Accepted: August 12, } 2018 \quad \text { Online Published: November 15, } 2018 \\ \text { doi:10.5539/jas.v10n12p148 } & \text { URL: https://doi.org/10.5539/jas.v10n12p148 }\end{array}$

\begin{abstract}
The objective of this study was to evaluate the agronomic traits of 80 accessions of elephant grass under the soil and weather conditions of Campos dos Goytacazes/RJ, Brazil. The experimental design was set as randomized blocks with 2 replicates. The experiment continued from March 2012 to May 2013, with 5 harvests made in the dry and rainy seasons. The following traits were assessed: percentage of dry matter (\%DM), dry matter yield (DMY), number of tillers per meter (NT), plant height (HGT), stem diameter (SD), leaf blade width (LBW) and leaf blade length (LBL). Data from each harvest were subjected to analysis of variance and to the Scott-Knott test $(\mathrm{P}<0.05)$. Tocher's optimization method, Mahalanobis distance, and canonical variables were utilized for the multiple traits, and the importance of the characters in the canonical variables. Genotypes with high yield were Elefante da Colômbia, Taiwan A-25, Albano, Hib. Gigante da Colômbia, Elefante de Pinda, Taiwan A-121, P241 Piracicaba, Guaçu/I.Z.2, CPAC, EMPASC 309, EMPASC 307, Australiano, and Pasto Panamá. Stem diameter (rainy season) and LBW (dry season) were the most important variables to differentiate between genotypes. There was wide phenotypic variation between genotypes, which could be divided into 15 groups by Tocher's optimization method.
\end{abstract}

Keywords: canonical variables, genetic divergence, Pennisetum purpureum

\section{Introduction}

Elephant grass (Pennisetum purpureum Schum.) is a tropical, perennial species with a high photosynthetic capacity. Because of its large genetic variability, it is able to adapt to the predominant climatic conditions throughout Brazil (Daher et al., 1997; Lista, 2008).

Introducing elephant grass germplasm has proved efficient in the identification of genotypes with desirable features (Shimoya et al., 2002; Lima et al., 2011) for future breeding programs. This demonstrates the importance of establishing and maintaining germplasm banks so as to guarantee the availability of the genetic variability necessary for breeding programs.

The elephant grass is a species of forage that has been highlighted by the nutritive balance and the healthy forage production per unit area (Ferreira et al., 2010). According to Santos et al. (2001) the protein contents in the elephant grass genotype, greater than $7 \%$. Levels lower than $7 \%$ are limiting to animal production due to digestibility coefficients and negative nitrogen balance. The chemical composition of elephantgrass is not limiting the digestion of non-ruminal fiber, which ends up reflecting not the animal's weight gain (Garcia et al., 2011).

Thus, genetic breeding programs for elephant grass aim to select material superior to cultivars used at present and adapted to environmental conditions in the different regions of the country, followed by post-breeding studies such as research on diversity based on molecular markers (Azevedo et al., 2012; Lima et al., 2011) and based on discriminating morphological traits. These studies guide the crossings, with a view to maximizing yield and quality and also enable the indication of genotypes for cultivation in the regions of interest.

The objective of this study was to estimate the diversity among 80 accessions of elephant grass through morphological traits and to identify the elite genotypes, for possible use in future breeding programs. 


\section{Materials and Methods}

\subsection{Establishment and Development of the Experiment}

The experiment was conducted on the Experimental Field of the State Center for Research on Agro-energy and Waste Use (Centro Estadual de Pesquisa em Agroenergia e Aproveitamento de Resíduos, Pesagro-RJ), located in

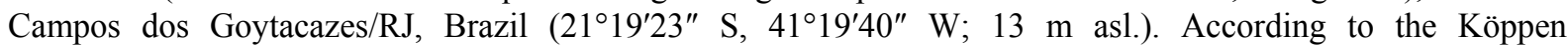
classification, the climate in the region is an Aw type (hot, wet tropical), with annual precipitation around 1,150 $\mathrm{mm}$. The soil was classified as a "Distrophic Argisol" (Embrapa, 2006), displaying the following properties: $\mathrm{pH}$ 5.5; phosphorus $\left(\mathrm{mg} \mathrm{dm}^{-3}\right)$ 18; potassium $\left(\mathrm{mg} \mathrm{dm}^{-3}\right)$ 83; $\mathrm{Ca}\left(\mathrm{cmol}_{\mathrm{c}} \mathrm{dm}^{-3}\right)$ 4.6; $\mathrm{Mg}\left(\mathrm{cmol}_{\mathrm{c}} \mathrm{dm}^{-3}\right) 3.0 ; \mathrm{Al}\left(\mathrm{cmol}_{\mathrm{c}} \mathrm{dm}^{-3}\right)$ $0.1 ; \mathrm{H}+\mathrm{Al}\left(\mathrm{cmol}_{\mathrm{c}} \mathrm{dm}^{-3}\right) 4.5$; and $\% \mathrm{C} 1.6$.

Eighty genotypes that belonged to the Active Germplasm Bank of Elephant Grass of Universidade Estadual do Norte Fluminense (UENF) were evaluated as shown in Table 1.

Table 1. Identification of 80 elephant grass genotypes (Campos dos Goytacazes, RJ, 2012/2013)

\begin{tabular}{|c|c|c|c|}
\hline Identification & Genotypes & Identification & Genotypes \\
\hline 1 & Elefante da Colômbia & 41 & Gramafante \\
\hline 2 & Mercker & 42 & Roxo \\
\hline 3 & Três Rios & 43 & Guaçu/I.Z.2 \\
\hline 4 & Napier Volta Grande & 44 & Cuba-115 \\
\hline 5 & Mercker Santa Rita & 45 & Cuba-116 \\
\hline 6 & Pusa Napier N 2 & 46 & Cuba-169 \\
\hline 7 & Gigante de Pinda & 47 & King Grass \\
\hline 8 & Napier N 2 & 48 & Roxo Botucatu \\
\hline 9 & Mercker S. E. A & 49 & Mineirão IPEACO \\
\hline 10 & Taiwan A-148 & 50 & Vruckwona Africano \\
\hline 11 & Porto Rico 534-B & 51 & Cameroon \\
\hline 12 & Taiwan A-25 & 52 & CPAC \\
\hline 13 & Albano & 53 & Guaçu \\
\hline 14 & Hib. Gigante Colômbia & 54 & Napierzinho \\
\hline 15 & Pusa Gigante Napier & 55 & IJ 7125 cv EMPASC 308 \\
\hline 16 & Elefante Híbrido 534-A & 56 & IJ 7126 cv EMPASC 310 \\
\hline 17 & Costa Rica & 57 & IJ $7127 \mathrm{cv}$ EMPASC 309 \\
\hline 18 & Cubano Pinda & 58 & IJ 7136 cv EMPASC 307 \\
\hline 19 & Mercker Pinda & 59 & IJ 7139 \\
\hline 20 & Mercker Pinda México & 60 & IJ $7141 \mathrm{cv}$ EMPASC 306 \\
\hline 21 & Mercker 86 México & 61 & Goiano \\
\hline 22 & Taiwan A-144 & 62 & CAC-262 \\
\hline 23 & Napier S.E.A. & 63 & Ibitinema \\
\hline 24 & Pusa Napier N 1 & 64 & 903-77 ou Australiano \\
\hline 25 & Elefante de Pinda & 65 & $13 \mathrm{AD}$ \\
\hline 26 & Mole de Volta Grande & 66 & $10 \mathrm{AD}$ IRI \\
\hline 27 & Napier & 67 & $07 \mathrm{AD}$ IRI \\
\hline 28 & Mercker Comum & 68 & Pasto Panamá \\
\hline 29 & Teresópolis & 69 & BAG-92 \\
\hline 30 & Taiwan A-46 & 70 & 09 AD IRI \\
\hline 31 & Duro de Volta Grande & 71 & $11 \mathrm{AD}$ IRI \\
\hline 32 & Turrialba & 72 & $06 \mathrm{AD}$ IRI \\
\hline 33 & Taiwan A-146 & 73 & $01 \mathrm{AD}$ IRI \\
\hline 34 & Cameroon - Piracicaba & 74 & 04 AD IRI \\
\hline 35 & Taiwan A-121 & 75 & $13 \mathrm{AD}$ IRI \\
\hline 36 & Vruckwona & 76 & $03 \mathrm{AD}$ IRI \\
\hline 37 & P241 Piracicaba & 77 & $02 \mathrm{AD}$ IRI \\
\hline 38 & IAC-Campinas & 78 & $08 \mathrm{AD}$ IRI \\
\hline 39 & Elefante C. Itapemirim & 79 & BAG UENF 79 \\
\hline 40 & Capim Cana D'África & 80 & BAG UENF 80 \\
\hline
\end{tabular}


Planting took place in February 2011, and 2 standardization cuts were made: in December 2011 and March 2012. After the second cut, 5 harvests were made during the evaluation phase: 2 in the dry season (June and August 2012), and 3 in the rainy season (October 2012, February and May 2013). A randomized-block experimental design was used with 2 replications. Each plot was $5.5 \times 2 \mathrm{~m}$, totaling $11 \mathrm{~m}^{2}$, with a floor area of $2 \mathrm{~m}^{2}$.

\subsection{Evaluated Traits}

At each harvest, the following morpho-agronomic traits were measured: plant height (HGT); stem diameter (SD); leaf blade width (LBW); and number of tillers (NT) per linear meter of row. To estimate dry matter yield (DMY) 2 plants from each plot were cut, placed in 5-kg paper bags and dried in a forced-ventilation oven at $65^{\circ} \mathrm{C}$ for 72 $\mathrm{h}$ until constant weight (air-dry). The dried material was subsequently ground in a Wiley mill with $1 \mathrm{~mm}$ screen, and then $2 \mathrm{~g}$ of ground material were weighed and dried in an oven at $105^{\circ} \mathrm{C}$ for $24 \mathrm{~h}$ (oven-dried sample). With the DMY data from the dry (Dry) and rainy (Rainy) seasons, it was possible to obtain the total dry matter yield (Total) and the percentage of total production from the dry season was calculated (\%Dry).

\subsection{Statistical Analyses}

For the univariate analyses, an analysis of variance (ANOVA) was performed for each variable in each evaluation, according to the following model: $Y_{i j}=M+G_{i}+B_{j}+e_{i j}$, where,

$Y_{i j}$ represents the observation of the $i$-th group in the $j$-th block;

$\mathrm{m}$ represents an overall constant associated with this random variable;

$\mathrm{G}_{\mathrm{i}}$ represents the effect of the i-th genotype;

$B_{j}$ represents the effect of the $j$-th block; and

$e_{i j}$ represents the experimental error associated with observation $Y$.

Subsequently, the means of the genotypes were grouped for each variable within each evaluation, using Scott and Knott's clustering. For the multivariate analyses, the canonical variables, relative importance of the traits in the canonical variables, generalized Mahalanobis distance (D2), and Tocher's clustering methods were employed using the GENES computer software (Cruz, 2013).

In the present study we addressed clustering through Tocher's optimization method by Mahalanobis distance (D2), which adopts the criterion that the average of the dissimilarity measures within each group should be lower than the distances between any groups. By the dissimilarity matrix we identified the pairs of most similar accessions, which formed the initial group, in which the possibility of inclusion of new accessions could be analyzed (Vasconcelos et al., 2011).

\section{Results and Discussion}

\subsection{Dry Matter Yield and Percentage of Dry Matter}

The overall mean for DMY in the 5 harvests was $23.92 \mathrm{t} / \mathrm{ha}$, with $45.6 \%$ produced during the dry season, and the remaining $54.4 \%$ produced during the rainy season (Table 2). Working with 132 hybrids from crossings involving 12 cultivars of millet and 11 cultivars of elephant grass, Souza Sobrinho et al. (2005) obtained an average DMY of $7.45 \mathrm{t} / \mathrm{ha}$ in 8 harvests. In a study in the region of Campos dos Goytacazes/RJ (Brazil), Lista (2008) evaluated the potential of 10 genotypes and 2 cultivars of elephant grass under irrigation and found an average of $15 \mathrm{t} / \mathrm{ha}$ in 4 harvests, while Leão et al. (2012) studied the performance of 13 genotypes of elephant grass and obtained an average of $4.86 \mathrm{t} / \mathrm{ha}$ in 2 harvests, value below that found in the present study.

Based on high DMY in wet and dry seasons and overall, the genotypes identified as being in the elite group were (1) Elefante da Colômbia, (12) Taiwan A-25, (13) Albano, (14) Hib. Gigante Colômbia, (25) Elefante de Pinda, (35) Taiwan A-121, (37) P241 Piracicaba, (43) Guaçu/I.Z.2, (52) CPAC, (57) EMPASC 309, (58) EMPASC 307, (64) Australiano and (68) Pasto Panamá. However, other genotypes were equally good in either wet or dry season and overall so they should not be dismissed.

The genotypes (3) Três Rios, (7) Gigante de Pinda, (22) Taiwan A-144, (26) Mole de Volta Grande, (27) Napier, (28) Mercker Comum, (31) Duro de Volta Grande, (33) Taiwan A-146, (37) P241 Piracicaba, (38) IAC-Campinas, (39) Elefante C. Itapemirim, (41) Gramafante, (42) Roxo, (44) Cuba-115, (45) Cuba-116, (46) Cuba-169, (47) King Grass, (49) Mineirão IPEACO, (53) Guaçu, (54) Napierzinho, (55) IJ 7125 cv EMPASC 308, (59) IJ 7139, (61) Goiano, (62) CAC-262, (65) 13 AD, (67) 07 AD IRI, (69) BAG - 92, (70) 09 AD IRI, (71) 11 AD IRI, (73) 01 AD IRI, (74) 04 AD IRI, (77) 02 AD IRI, (78) 08 AD IRI showed higher DMY in the dry season, however, lower DMY in the rainy season compared to the other evaluated genotypes. According to Cunha et al. (2013) it is important to identify clones of elephant grass that show superiority in productive 
performance and greater stability in forage production throughout the year, which in this case are part of the group of elite genotypes.

Table 2. Dry matter yields (DMY), in t/ha, of 80 genotypes of elephant grass in the dry and rainy seasons and total, plus percentage produced during the dry season (\%Dry), in five harvests (Campos dos Goytacazes/RJ, Brazil, 2012/2013)

\begin{tabular}{|c|c|c|c|c|c|c|c|c|c|}
\hline Genotype & Dry & Rainy & Total & \%Dry & $\mathrm{G}^{1}$ & Dry & Rainy & Total & \%Dry \\
\hline 1 & $11.79 \mathrm{a}^{1}$ & $19.61 \mathrm{a}$ & $31.40 \mathrm{a}$ & $37.59 \mathrm{~b}$ & 41 & $16.89 \mathrm{a}$ & $12.26 \mathrm{~b}$ & $29.15 \mathrm{a}$ & $58.27 \mathrm{a}$ \\
\hline 2 & $8.93 \mathrm{~b}$ & $11.26 \mathrm{~b}$ & $20.19 b$ & $43.46 \mathrm{~b}$ & 42 & $11.53 \mathrm{a}$ & $8.52 \mathrm{~b}$ & $20.06 \mathrm{~b}$ & $57.50 \mathrm{a}$ \\
\hline 3 & $12.28 \mathrm{a}$ & $9.76 \mathrm{~b}$ & $22.04 \mathrm{~b}$ & $55.87 \mathrm{a}$ & 43 & $14.66 \mathrm{a}$ & $19.05 \mathrm{a}$ & $33.71 \mathrm{a}$ & $45.55 \mathrm{a}$ \\
\hline 4 & $8.22 \mathrm{~b}$ & $7.99 \mathrm{~b}$ & $16.22 \mathrm{~b}$ & $50.34 \mathrm{a}$ & 44 & $15.20 \mathrm{a}$ & $11.52 \mathrm{~b}$ & $26.73 \mathrm{a}$ & $56.68 \mathrm{a}$ \\
\hline 5 & $7.31 \mathrm{~b}$ & $17.46 \mathrm{a}$ & $24.77 \mathrm{a}$ & $30.35 \mathrm{~b}$ & 45 & $10.86 \mathrm{a}$ & $10.76 \mathrm{~b}$ & $21.62 \mathrm{~b}$ & $50.92 \mathrm{a}$ \\
\hline 6 & $6.58 \mathrm{~b}$ & $9.00 \mathrm{~b}$ & $15.58 \mathrm{~b}$ & $42.99 \mathrm{~b}$ & 46 & $12.63 \mathrm{a}$ & $11.44 \mathrm{~b}$ & $24.06 \mathrm{a}$ & $53.22 \mathrm{a}$ \\
\hline 7 & $14.93 \mathrm{a}$ & $12.38 \mathrm{~b}$ & $27.32 \mathrm{a}$ & $52.65 \mathrm{a}$ & 47 & $11.80 \mathrm{a}$ & $13.97 \mathrm{~b}$ & $25.78 \mathrm{a}$ & $47.65 \mathrm{a}$ \\
\hline 8 & $62.17 \mathrm{~b}$ & $12.95 \mathrm{~b}$ & $19.17 \mathrm{~b}$ & $32.45 \mathrm{~b}$ & 48 & $7.96 \mathrm{~b}$ & $10.90 \mathrm{~b}$ & $18.86 \mathrm{~b}$ & $42.08 \mathrm{~b}$ \\
\hline 9 & $8.01 \mathrm{~b}$ & $13.43 \mathrm{~b}$ & $21.44 \mathrm{~b}$ & $37.08 \mathrm{~b}$ & 49 & $12.05 \mathrm{a}$ & $12.79 \mathrm{~b}$ & $24.83 \mathrm{a}$ & $48.16 \mathrm{a}$ \\
\hline 10 & $8.61 \mathrm{~b}$ & $17.53 \mathrm{a}$ & $26.15 \mathrm{a}$ & $32.75 \mathrm{~b}$ & 50 & $9.91 \mathrm{~b}$ & $17.76 \mathrm{a}$ & $27.67 \mathrm{a}$ & $35.22 \mathrm{~b}$ \\
\hline 11 & $8.59 \mathrm{~b}$ & $7.80 \mathrm{~b}$ & $16.39 \mathrm{~b}$ & $52.53 \mathrm{a}$ & 51 & $9.39 \mathrm{~b}$ & $9.87 \mathrm{~b}$ & $19.26 \mathrm{~b}$ & $4.932 \mathrm{a}$ \\
\hline 12 & $11.28 \mathrm{a}$ & $14.73 \mathrm{a}$ & $26.01 \mathrm{a}$ & $42.06 \mathrm{~b}$ & 52 & $12.53 \mathrm{a}$ & $14.93 \mathrm{a}$ & $27.46 \mathrm{a}$ & $45.43 \mathrm{a}$ \\
\hline 13 & $10.87 \mathrm{a}$ & $14.91 \mathrm{a}$ & $25.78 \mathrm{a}$ & $42.06 \mathrm{~b}$ & 53 & $11.68 \mathrm{a}$ & $10.60 \mathrm{~b}$ & $22.29 \mathrm{~b}$ & $51.72 \mathrm{a}$ \\
\hline 14 & $11.19 \mathrm{a}$ & $17.18 \mathrm{a}$ & $28.37 \mathrm{a}$ & $40.33 \mathrm{~b}$ & 54 & $11.23 \mathrm{a}$ & $10.37 \mathrm{~b}$ & $21.60 \mathrm{~b}$ & $52.60 \mathrm{a}$ \\
\hline 15 & $9.69 \mathrm{~b}$ & $12.37 \mathrm{~b}$ & $22.06 \mathrm{~b}$ & $43.75 \mathrm{a}$ & 55 & $11.60 \mathrm{a}$ & $14.03 \mathrm{~b}$ & $25.63 \mathrm{a}$ & $45.31 \mathrm{a}$ \\
\hline 16 & $9.65 \mathrm{~b}$ & $11.86 \mathrm{~b}$ & $21.51 \mathrm{~b}$ & $45.07 \mathrm{a}$ & 56 & $8.06 \mathrm{~b}$ & $15.15 \mathrm{a}$ & $23.21 \mathrm{~b}$ & $35.16 \mathrm{~b}$ \\
\hline 17 & $7.55 \mathrm{~b}$ & $12.37 \mathrm{~b}$ & $19.92 \mathrm{~b}$ & $37.76 \mathrm{~b}$ & 57 & $14.95 \mathrm{a}$ & $16.44 \mathrm{a}$ & $31.39 \mathrm{a}$ & $47.69 \mathrm{a}$ \\
\hline 18 & $6.01 \mathrm{~b}$ & $6.50 \mathrm{~b}$ & $12.51 \mathrm{~b}$ & $48.05 \mathrm{a}$ & 58 & $16.64 \mathrm{a}$ & $18.67 \mathrm{a}$ & $35.31 \mathrm{a}$ & $47.05 \mathrm{a}$ \\
\hline 19 & $5.90 \mathrm{~b}$ & $11.83 \mathrm{~b}$ & $17.74 b$ & $33.29 \mathrm{~b}$ & 59 & $12.95 \mathrm{a}$ & $11.61 \mathrm{~b}$ & $24.56 \mathrm{a}$ & $53.17 \mathrm{a}$ \\
\hline 20 & $5.58 \mathrm{~b}$ & $10.54 \mathrm{~b}$ & $16.12 \mathrm{~b}$ & $34.10 \mathrm{~b}$ & 60 & $9.41 \mathrm{~b}$ & $10.93 \mathrm{~b}$ & $20.34 \mathrm{~b}$ & $46.16 \mathrm{a}$ \\
\hline 21 & $8.661 \mathrm{~b}$ & $13.93 \mathrm{~b}$ & $22.59 \mathrm{~b}$ & $38.17 \mathrm{~b}$ & 61 & $11.63 \mathrm{a}$ & $13.04 \mathrm{~b}$ & $24.68 \mathrm{a}$ & $47.64 \mathrm{a}$ \\
\hline 22 & $11.77 \mathrm{a}$ & $13.63 \mathrm{~b}$ & $25.40 \mathrm{a}$ & $46.61 \mathrm{a}$ & 62 & $13.81 \mathrm{a}$ & $13.61 \mathrm{~b}$ & $27.42 \mathrm{a}$ & $50.35 \mathrm{a}$ \\
\hline 23 & $6.35 \mathrm{~b}$ & $16.45 \mathrm{a}$ & $22.80 \mathrm{~b}$ & $28.20 \mathrm{~b}$ & 63 & $9.65 \mathrm{~b}$ & $13.89 \mathrm{~b}$ & $23.55 \mathrm{~b}$ & $41.48 \mathrm{~b}$ \\
\hline 24 & $9.29 \mathrm{~b}$ & $12.35 \mathrm{~b}$ & $21.64 \mathrm{~b}$ & $44.41 \mathrm{a}$ & 64 & $14.89 \mathrm{a}$ & $14.58 \mathrm{a}$ & $29.48 \mathrm{a}$ & $50.20 \mathrm{a}$ \\
\hline 25 & $14.95 \mathrm{a}$ & $16.00 \mathrm{a}$ & $30.95 \mathrm{a}$ & $47.42 \mathrm{a}$ & 65 & $12.55 \mathrm{a}$ & $12.64 \mathrm{~b}$ & $25.18 \mathrm{a}$ & $43.07 \mathrm{~b}$ \\
\hline 26 & $12.29 \mathrm{a}$ & $12.21 \mathrm{~b}$ & $24.49 \mathrm{a}$ & $48.44 \mathrm{a}$ & 66 & $10.02 \mathrm{~b}$ & $12.84 \mathrm{~b}$ & $22.86 \mathrm{~b}$ & $52.91 \mathrm{a}$ \\
\hline 27 & $10.96 \mathrm{a}$ & $10.07 \mathrm{~b}$ & $21.03 \mathrm{~b}$ & $49.79 \mathrm{a}$ & 67 & $12.19 \mathrm{a}$ & $11.02 \mathrm{~b}$ & $23.21 \mathrm{~b}$ & $47.58 \mathrm{a}$ \\
\hline 28 & $11.40 \mathrm{a}$ & $9.07 \mathrm{~b}$ & $20.47 \mathrm{~b}$ & $55.45 \mathrm{a}$ & 68 & $20.05 \mathrm{a}$ & $22.14 \mathrm{a}$ & $42.19 \mathrm{a}$ & $56.57 \mathrm{a}$ \\
\hline 29 & $8.66 \mathrm{~b}$ & $15.74 \mathrm{a}$ & $24.40 \mathrm{a}$ & $34.99 \mathrm{~b}$ & 69 & $14.03 \mathrm{a}$ & $10.67 \mathrm{~b}$ & $24.70 \mathrm{a}$ & $50.44 \mathrm{a}$ \\
\hline 30 & $8.46 \mathrm{~b}$ & $10.33 \mathrm{~b}$ & $18.79 \mathrm{~b}$ & $46.04 \mathrm{a}$ & 70 & $13.75 \mathrm{a}$ & $134.73 b$ & $27.22 \mathrm{a}$ & $48.61 \mathrm{a}$ \\
\hline 31 & $13.17 \mathrm{a}$ & $13.87 \mathrm{~b}$ & $27.05 \mathrm{a}$ & $50.21 \mathrm{a}$ & 71 & $11.42 \mathrm{a}$ & $120.87 b$ & $23.51 \mathrm{~b}$ & $30.78 \mathrm{~b}$ \\
\hline 32 & $10.59 \mathrm{~b}$ & $14.38 \mathrm{a}$ & $24.99 \mathrm{a}$ & $42.29 \mathrm{~b}$ & 72 & $5.85 \mathrm{~b}$ & $13.11 \mathrm{~b}$ & $18.96 \mathrm{~b}$ & $55.72 \mathrm{a}$ \\
\hline 33 & $12.89 \mathrm{a}$ & $11.56 \mathrm{~b}$ & $24.46 \mathrm{a}$ & $52.39 \mathrm{a}$ & 73 & $12.04 \mathrm{a}$ & $96.93 \mathrm{~b}$ & $21.73 \mathrm{~b}$ & $48.65 \mathrm{a}$ \\
\hline 34 & $8.77 \mathrm{~b}$ & $10.30 \mathrm{~b}$ & $19.07 \mathrm{~b}$ & $45.98 \mathrm{a}$ & 74 & $12.06 \mathrm{a}$ & $12.75 \mathrm{~b}$ & $24.81 \mathrm{a}$ & $39.81 \mathrm{~b}$ \\
\hline 35 & $11.79 \mathrm{a}$ & $14.71 \mathrm{a}$ & $26.49 \mathrm{a}$ & $44.57 \mathrm{a}$ & 75 & $8.71 \mathrm{~b}$ & $13.02 \mathrm{~b}$ & $21.73 b$ & $29.33 \mathrm{~b}$ \\
\hline 36 & $7.56 \mathrm{~b}$ & $7.84 \mathrm{~b}$ & $15.39 \mathrm{~b}$ & $48.94 \mathrm{a}$ & 76 & $7.77 \mathrm{~b}$ & $18.88 \mathrm{a}$ & $26.66 \mathrm{a}$ & $51.03 \mathrm{a}$ \\
\hline 37 & $12.78 \mathrm{a}$ & $17.92 \mathrm{~b}$ & $30.70 \mathrm{a}$ & $41.13 \mathrm{~b}$ & 77 & $15.68 \mathrm{a}$ & $13.37 \mathrm{~b}$ & $29.05 \mathrm{a}$ & $52.86 \mathrm{a}$ \\
\hline 38 & $13.34 \mathrm{a}$ & $12.70 \mathrm{~b}$ & $26.05 \mathrm{a}$ & $51.27 \mathrm{a}$ & 78 & $15.03 \mathrm{a}$ & $12.39 \mathrm{~b}$ & $27.41 \mathrm{a}$ & $41.13 \mathrm{~b}$ \\
\hline 39 & $13.33 \mathrm{a}$ & $13.25 \mathrm{~b}$ & $26.58 \mathrm{a}$ & $49.14 \mathrm{a}$ & 79 & $7.84 \mathrm{~b}$ & $11.31 \mathrm{~b}$ & $19.15 \mathrm{~b}$ & $44.25 \mathrm{a}$ \\
\hline 40 & $9.58 \mathrm{~b}$ & $10.63 \mathrm{~b}$ & $20.22 \mathrm{~b}$ & $47.40 \mathrm{a}$ & 80 & $9.98 \mathrm{~b}$ & $12.46 \mathrm{~b}$ & $22.44 \mathrm{~b}$ & $50.79 a$ \\
\hline Mean & 10.96 & 12.96 & 23.92 & 45.62 & & & & & \\
\hline $\mathrm{CV}(\%)$ & 23.21 & 21.37 & 17.47 & 15.83 & & & & & \\
\hline
\end{tabular}

Note. ${ }^{1}$ Values within columns followed by the same letter do not differ by Scott-Knott's test at $5 \%$ probability.

The number of tillers per linear meter of row (NT) varied between 22.0 and 91.3 for genotypes Capim Cana D'África and BAG-92, respectively, and averaged 45.1 in the dry season. In the rainy season, NT varied from 26.1 to 72.6 for genotypes Goiano and EMPASC 309, respectively, averaging 46.0 (Table 2); the aforementioned values are higher than the 21.6 tillers found by Daher et al. (1997). Silva (2011) observed an average of 47.6 and Cunha (2012) obtained average values varying from 27.7 to 46.8 tillers. However, Daher et al. (2004) stressed that 
this variable was the to the most useful for predicting dry matter yield potential, being positively correlated with this parameter.

Plant height averaged 1.43 and $1.22 \mathrm{~m}$ in the dry and rainy seasons, respectively (Table 3 ). Lower HGT values are found in studies with shorter cutting intervals (Cavalcante \& Lira, 2010). Leão et al. (2012) found an average HGT for the genotypes of $1.57 \mathrm{~m}$, which exceeds the value obtained in the current study.

Table 3. Total means for number of tillers (NT) per linear meter and plant height (HGT, m), of 80 genotypes of elephant grass in the dry and rainy seasons, in five harvests (Campos dos Goytacazes/RJ, Brazil, 2012/2013)

\begin{tabular}{|c|c|c|c|c|c|c|c|c|c|c|c|c|c|c|c|c|c|}
\hline \multirow{2}{*}{$\mathrm{G}^{1}$} & \multicolumn{4}{|c|}{ NT } & \multicolumn{4}{|c|}{ HGT } & \multirow{2}{*}{$\mathrm{G}^{1}$} & \multicolumn{4}{|c|}{ NT } & \multicolumn{4}{|c|}{ HGT } \\
\hline & Dry & & Rainy & & Dry & & Rainy & & & Dry & & Rainy & & Dry & & Rainy & \\
\hline 1 & 52.57 & $\mathrm{a}^{1}$ & 52.10 & $\mathrm{a}$ & 1.57 & $\mathrm{a}$ & 1.41 & $\mathrm{a}$ & 41 & 53.28 & $\mathrm{a}$ & 41.23 & $\mathrm{a}$ & 1.64 & $\mathrm{a}$ & 1.31 & $\bar{a}$ \\
\hline 2 & 36.32 & $\mathrm{~b}$ & 39.42 & $\mathrm{a}$ & 1.30 & $\mathrm{~b}$ & 1.22 & $\mathrm{a}$ & 42 & 38.25 & $\mathrm{~b}$ & 32.50 & $\mathrm{a}$ & 1.64 & $\mathrm{a}$ & 1.39 & a \\
\hline 3 & 51.61 & $\mathrm{a}$ & 52.25 & $\mathrm{a}$ & 1.21 & $\mathrm{~b}$ & 1.13 & $\mathrm{~b}$ & 43 & 45.33 & b & 70.43 & $\mathrm{a}$ & 1.45 & $\mathrm{a}$ & 1.16 & b \\
\hline 4 & 51.25 & $\mathrm{a}$ & 38.65 & $\mathrm{a}$ & 1.13 & $\mathrm{~b}$ & 1.03 & $b$ & 44 & 47.50 & $\mathrm{~b}$ & 45.10 & $\mathrm{a}$ & 1.69 & $\mathrm{a}$ & 1.29 & $\mathrm{a}$ \\
\hline 5 & 43.25 & $\mathrm{~b}$ & 60.83 & $\mathrm{a}$ & 1.26 & $\mathrm{~b}$ & 1.26 & $\mathrm{a}$ & 45 & 41.75 & $\mathrm{~b}$ & 32.59 & $\mathrm{a}$ & 1.79 & $\mathrm{a}$ & 1.27 & $\mathrm{a}$ \\
\hline 6 & 44.67 & $\mathrm{~b}$ & 35.39 & $\mathrm{a}$ & 1.22 & $\mathrm{~b}$ & 1.22 & $\mathrm{a}$ & 46 & 26.88 & $\mathrm{~b}$ & 46.08 & $\mathrm{a}$ & 1.67 & $\mathrm{a}$ & 1.19 & b \\
\hline 7 & 75.45 & $\mathrm{a}$ & 45.45 & $\mathrm{a}$ & 1.30 & $\mathrm{~b}$ & 1.20 & $b$ & 47 & 46.95 & $\mathrm{~b}$ & 43.88 & $\mathrm{a}$ & 1.59 & $\mathrm{a}$ & 1.28 & $\mathrm{a}$ \\
\hline 8 & 35.25 & $\mathrm{~b}$ & 50.19 & $\mathrm{a}$ & 1.22 & $\mathrm{~b}$ & 1.26 & $\mathrm{a}$ & 48 & 22.61 & $\mathrm{~b}$ & 40.21 & $\mathrm{a}$ & 1.67 & $\mathrm{a}$ & 1.08 & $\mathrm{~b}$ \\
\hline 9 & 36.00 & $\mathrm{~b}$ & 47.29 & $\mathrm{a}$ & 1.26 & $\mathrm{~b}$ & 1.25 & $\mathrm{a}$ & 49 & 45.58 & b & 41.49 & $\mathrm{a}$ & 1.42 & $\mathrm{a}$ & 1.17 & b \\
\hline 10 & 42.75 & $\mathrm{~b}$ & 47.17 & $\mathrm{a}$ & 1.44 & $\mathrm{a}$ & 1.28 & $\mathrm{a}$ & 50 & 45.50 & $\mathrm{~b}$ & 44.67 & $\mathrm{a}$ & 1.50 & $\mathrm{a}$ & 1.34 & $\mathrm{a}$ \\
\hline 11 & 45.17 & $\mathrm{~b}$ & 48.00 & $\mathrm{a}$ & 1.48 & $\mathrm{a}$ & 1.13 & $\mathrm{~b}$ & 51 & 25.50 & $\mathrm{~b}$ & 36.88 & $\mathrm{a}$ & 1.62 & $\mathrm{a}$ & 1.26 & $\mathrm{a}$ \\
\hline 12 & 47.09 & $\mathrm{~b}$ & 46.06 & $\mathrm{a}$ & 1.33 & $\mathrm{~b}$ & 1.25 & $\mathrm{a}$ & 52 & 26.30 & $b$ & 50.04 & $\mathrm{a}$ & 1.60 & $\mathrm{a}$ & 1.23 & $\mathrm{a}$ \\
\hline 13 & 25.31 & $\mathrm{~b}$ & 28.97 & $\mathrm{a}$ & 1.61 & $\mathrm{a}$ & 1.39 & $\mathrm{a}$ & 53 & 31.63 & b & 50.12 & $\mathrm{a}$ & 1.39 & $\mathrm{~b}$ & 1.18 & b \\
\hline 14 & 45.20 & $\mathrm{~b}$ & 50.14 & $\mathrm{a}$ & 1.50 & $\mathrm{a}$ & 1.32 & $\mathrm{a}$ & 54 & 41.21 & $\mathrm{~b}$ & 52.95 & $\mathrm{a}$ & 1.47 & $\mathrm{a}$ & 1.28 & $\mathrm{a}$ \\
\hline 15 & 37.75 & $\mathrm{~b}$ & 43.46 & $\mathrm{a}$ & 1.39 & $\mathrm{~b}$ & 1.39 & $\mathrm{a}$ & 55 & 51.25 & $\mathrm{a}$ & 44.39 & $\mathrm{a}$ & 1.38 & $\mathrm{~b}$ & 1.25 & $\mathrm{a}$ \\
\hline 16 & 28.14 & $\mathrm{~b}$ & 33.56 & $\mathrm{a}$ & 1.30 & $\mathrm{~b}$ & 1.30 & $\mathrm{a}$ & 56 & 39.51 & $\mathrm{~b}$ & 36.62 & $\mathrm{a}$ & 1.41 & $\mathrm{~b}$ & 1.27 & $\mathrm{a}$ \\
\hline 17 & 26.50 & $\mathrm{~b}$ & 45.37 & $\mathrm{a}$ & 1.17 & $\mathrm{~b}$ & 1.21 & $\mathrm{a}$ & 57 & 63.45 & $\mathrm{a}$ & 72.64 & $\mathrm{a}$ & 1.45 & $\mathrm{a}$ & 1.26 & $\mathrm{a}$ \\
\hline 18 & 24.71 & $\mathrm{~b}$ & 37.97 & $\mathrm{a}$ & 1.27 & $\mathrm{~b}$ & 1.29 & $\mathrm{a}$ & 58 & 63.25 & $\mathrm{a}$ & 57.39 & $\mathrm{a}$ & 1.53 & $\mathrm{a}$ & 1.25 & $\mathrm{a}$ \\
\hline 19 & 43.75 & $\mathrm{~b}$ & 40.06 & $\mathrm{a}$ & 1.11 & $\mathrm{~b}$ & 1.22 & $\mathrm{a}$ & 59 & 31.50 & b & 32.75 & $\mathrm{a}$ & 1.80 & $\mathrm{a}$ & 1.31 & $\mathrm{a}$ \\
\hline 20 & 32.25 & $\mathrm{~b}$ & 33.79 & $\mathrm{a}$ & 1.22 & $\mathrm{~b}$ & 1.17 & $b$ & 60 & 30.00 & $\mathrm{~b}$ & 43.84 & $\mathrm{a}$ & 1.54 & $\mathrm{a}$ & 1.21 & $\mathrm{~b}$ \\
\hline 21 & 41.00 & $\mathrm{~b}$ & 41.05 & $\mathrm{a}$ & 1.22 & $\mathrm{~b}$ & 1.17 & $b$ & 61 & 40.45 & $\mathrm{~b}$ & 26.05 & $\mathrm{a}$ & 1.58 & $\mathrm{a}$ & 1.25 & $\mathrm{a}$ \\
\hline 22 & 42.25 & $\mathrm{~b}$ & 45.33 & $\mathrm{a}$ & 1.52 & $\mathrm{a}$ & 1.25 & $\mathrm{a}$ & 62 & 63.25 & $\mathrm{a}$ & 52.77 & $\mathrm{a}$ & 1.48 & $\mathrm{a}$ & 1.25 & $\mathrm{a}$ \\
\hline 23 & 40.25 & $\mathrm{~b}$ & 49.24 & $\mathrm{a}$ & 1.43 & $\mathrm{a}$ & 1.25 & $\mathrm{a}$ & 63 & 37.26 & $\mathrm{~b}$ & 43.02 & $\mathrm{a}$ & 1.46 & $\mathrm{a}$ & 1.05 & $\mathrm{~b}$ \\
\hline 24 & 36.75 & $\mathrm{~b}$ & 31.89 & $\mathrm{a}$ & 1.34 & $\mathrm{~b}$ & 1.21 & $b$ & 64 & 74.42 & $\mathrm{a}$ & 58.67 & $\mathrm{a}$ & 1.53 & $\mathrm{a}$ & 1.24 & $\mathrm{a}$ \\
\hline 25 & 57.48 & $\mathrm{a}$ & 40.56 & $\mathrm{a}$ & 1.37 & $\mathrm{~b}$ & 1.29 & $\mathrm{a}$ & 65 & 72.50 & $\mathrm{a}$ & 51.81 & $\mathrm{a}$ & 1.34 & $\mathrm{~b}$ & 1.20 & $\mathrm{~b}$ \\
\hline 26 & 60.75 & $\mathrm{a}$ & 69.45 & $\mathrm{a}$ & 1.25 & $\mathrm{~b}$ & 1.23 & $\mathrm{a}$ & 66 & 38.50 & $\mathrm{~b}$ & 45.91 & $\mathrm{a}$ & 1.63 & $\mathrm{a}$ & 1.16 & $\mathrm{~b}$ \\
\hline 27 & 50.51 & $\mathrm{a}$ & 56.56 & $\mathrm{a}$ & 1.17 & $\mathrm{~b}$ & 1.15 & $b$ & 67 & 54.70 & $\mathrm{a}$ & 46.14 & $\mathrm{a}$ & 1.19 & $\mathrm{~b}$ & 1.04 & $\mathrm{~b}$ \\
\hline 28 & 51.59 & $\mathrm{a}$ & 56.22 & $\mathrm{a}$ & 1.17 & $\mathrm{~b}$ & 1.05 & $\mathrm{~b}$ & 68 & 67.59 & $\mathrm{a}$ & 49.34 & $\mathrm{a}$ & 1.60 & $\mathrm{a}$ & 1.46 & $\mathrm{a}$ \\
\hline 29 & 44.00 & $\mathrm{~b}$ & 62.83 & $\mathrm{a}$ & 1.58 & $\mathrm{a}$ & 1.16 & $\mathrm{~b}$ & 69 & 91.25 & $\mathrm{a}$ & 64.56 & $\mathrm{a}$ & 1.30 & $\mathrm{~b}$ & 1.03 & $\mathrm{~b}$ \\
\hline 30 & 42.25 & $\mathrm{~b}$ & 47.53 & $\mathrm{a}$ & 1.60 & $\mathrm{a}$ & 1.23 & $\mathrm{a}$ & 70 & 44.50 & $\mathrm{~b}$ & 59.08 & $\mathrm{a}$ & 1.49 & $\mathrm{a}$ & 1.15 & b \\
\hline 31 & 45.13 & $\mathrm{~b}$ & 49.88 & $\mathrm{a}$ & 1.52 & $\mathrm{a}$ & 1.17 & $\mathrm{~b}$ & 71 & 55.05 & $\mathrm{a}$ & 43.75 & $\mathrm{a}$ & 1.19 & $\mathrm{~b}$ & 1.13 & b \\
\hline 32 & 33.44 & $\mathrm{~b}$ & 35.62 & $\mathrm{a}$ & 1.53 & $\mathrm{a}$ & 1.32 & $\mathrm{a}$ & 72 & 49.92 & $\mathrm{a}$ & 55.92 & $\mathrm{a}$ & 1.27 & $\mathrm{~b}$ & 1.09 & $\mathrm{~b}$ \\
\hline 33 & 63.51 & $\mathrm{a}$ & 43.47 & $\mathrm{a}$ & 1.30 & $\mathrm{~b}$ & 1.14 & $\mathrm{~b}$ & 73 & 78.61 & $\mathrm{a}$ & 30.17 & $\mathrm{a}$ & 1.31 & $\mathrm{~b}$ & 1.13 & $\mathrm{~b}$ \\
\hline 34 & 49.35 & $\mathrm{a}$ & 40.14 & $\mathrm{a}$ & 1.50 & $\mathrm{a}$ & 1.31 & $\mathrm{a}$ & 74 & 48.44 & b & 37.17 & $\mathrm{a}$ & 1.34 & $\mathrm{~b}$ & 1.13 & b \\
\hline 35 & 53.28 & $\mathrm{a}$ & 38.83 & $\mathrm{a}$ & 1.06 & $\mathrm{~b}$ & 1.10 & $\mathrm{~b}$ & 75 & 40.53 & $\mathrm{~b}$ & 55.14 & $\mathrm{a}$ & 1.31 & $\mathrm{~b}$ & 1.16 & $\mathrm{~b}$ \\
\hline 36 & 31.31 & $\mathrm{~b}$ & 27.79 & $\mathrm{a}$ & 1.33 & $\mathrm{~b}$ & 1.28 & $\mathrm{a}$ & 76 & 31.25 & $\mathrm{~b}$ & 51.24 & $\mathrm{a}$ & 1.37 & $\mathrm{~b}$ & 1.11 & $\mathrm{~b}$ \\
\hline 37 & 32.07 & $\mathrm{~b}$ & 62.00 & $\mathrm{a}$ & 1.31 & $\mathrm{~b}$ & 1.04 & $\mathrm{~b}$ & 77 & 56.75 & $\mathrm{a}$ & 57.05 & $\mathrm{a}$ & 1.47 & $\mathrm{a}$ & 1.20 & b \\
\hline 38. & 34.47 & $\mathrm{~b}$ & 48.00 & $\mathrm{a}$ & 1.40 & $b$ & 1.23 & $\mathrm{a}$ & 78 & 52.25 & $\mathrm{a}$ & 46.80 & $\mathrm{a}$ & 1.35 & $\mathrm{~b}$ & 1.16 & b \\
\hline 39 & 52.25 & $\mathrm{a}$ & 58.67 & $\mathrm{a}$ & 1.72 & $\mathrm{a}$ & 1.27 & $\mathrm{a}$ & 79 & 43.25 & $\mathrm{~b}$ & 46.25 & $\mathrm{a}$ & 1.26 & $\mathrm{~b}$ & 1.11 & $\mathrm{~b}$ \\
\hline 40 & 22.00 & $\mathrm{~b}$ & 31.24 & $\mathrm{a}$ & 1.77 & $\mathrm{a}$ & 1.46 & $\mathrm{a}$ & 80 & 39.75 & b & 32.72 & $\mathrm{a}$ & 1.85 & $\mathrm{a}$ & 1.15 & b \\
\hline Mean Dry & 45.09 & & & & 1.43 & & & & Mean Rainy & 46.02 & & & & 1.22 & & & \\
\hline
\end{tabular}

Note ${ }^{1}$ Values within columns followed by the same letter do not differ by Scott-Knott's test at $5 \%$ probability. 
Stem diameter in the dry season averaged $1.06 \mathrm{~cm}$ and ranged from $0.70 \mathrm{~cm}$ for Mercker Comum to $1.45 \mathrm{~cm}$ for Pusa Napier and $1.43 \mathrm{~cm}$ for Albano. In the rainy season, the average stem diameter was $1.09 \mathrm{~cm}$ and again ranged from $0.67 \mathrm{~cm}$ for Mercker Comum to $1.45 \mathrm{~cm}$ for Albano and $1.43 \mathrm{~cm}$ for Pusa Napier (Table 4). Studying dwarf elephant grass, Silva et al. (2009) obtained an average of $1.05 \mathrm{~cm}$; similarly Silva (2011) obtained $1.66 \mathrm{~cm}$, and Cunha (2012) found higher stem diameters: 1.03 and $1.64 \mathrm{~cm}$, demonstrating the large variability in this trait among the evaluated accessions. Rezende et al. (2008) evaluated Cameroon grass in the dry season, and obtained stem diameters varying from 0.54 to $1.51 \mathrm{~cm}$, and 1.59 to $2.03 \mathrm{~cm}$ in the rainy season.

The average LBWs were 2.64 and $2.69 \mathrm{~cm}$ in the dry and rainy seasons, respectively (Table 4). Zhang et al. (2010) obtained an average of $3.33 \mathrm{~cm}$, and Silva (2011) obtained $3.11 \mathrm{~cm}$, which are higher values than those found in the present study. However, Silva et al. (2009) found an average value lower than $1.96 \mathrm{~cm}$.

Table 4. Total means for stem diameter (SD, $\mathrm{cm}$ ) and leaf blade width (LBW, $\mathrm{cm}$ ) of 80 genotypes of elephant grass in the dry and rainy seasons, in five harvests (Campos dos Goytacazes/RJ, Brazil, 2012/2013)

\begin{tabular}{|c|c|c|c|c|c|c|c|c|c|c|c|c|c|c|c|c|c|}
\hline \multirow{2}{*}{$\mathrm{G}^{1}$} & \multicolumn{4}{|c|}{ SD } & \multicolumn{4}{|c|}{ LBW } & \multirow{2}{*}{$\mathrm{G}^{1}$} & \multicolumn{4}{|c|}{ SD } & \multicolumn{4}{|c|}{ LBW } \\
\hline & Dry & & Rainy & & Dry & & Rainy & & & Dry & & Rainy & & Dry & & Rainy & \\
\hline 1 & 1.13 & $\mathrm{a}$ & 1.12 & $\begin{array}{lc}\mathrm{c} \\
\end{array}$ & 2.38 & $\mathrm{~b}$ & 3.07 & $\mathrm{a}$ & 41 & 1.00 & $\mathrm{~b}$ & 0.99 & $\mathrm{~d}$ & 2.78 & $\mathrm{a}$ & 3.01 & $\mathrm{a}$ \\
\hline 2 & 1.25 & $\mathrm{a}$ & 1.30 & $\mathrm{~b}$ & 2.79 & $\mathrm{a}$ & 3.18 & $\mathrm{a}$ & 42 & 1.09 & $\mathrm{~b}$ & 1.10 & c & 2.46 & $\mathrm{~b}$ & 2.76 & $\mathrm{~b}$ \\
\hline 3 & 1.13 & $\mathrm{a}$ & 1.18 & $\mathrm{c}$ & 2.27 & $\mathrm{~b}$ & 2.87 & $\mathrm{a}$ & 43 & 1.13 & $\mathrm{a}$ & 1.19 & $\mathrm{c}$ & 3.03 & $\mathrm{a}$ & 3.05 & $\mathrm{a}$ \\
\hline 4 & 1.13 & $\mathrm{a}$ & 1.15 & c & 3.13 & $\mathrm{a}$ & 3.37 & $\mathrm{a}$ & 44 & 1.08 & $\mathrm{~b}$ & 1.17 & c & 2.53 & $\mathrm{~b}$ & 2.93 & $\mathrm{a}$ \\
\hline 5 & 1.31 & $\mathrm{a}$ & 1.25 & $\mathrm{~b}$ & 2.63 & $\mathrm{~b}$ & 3.01 & $\mathrm{a}$ & 45 & 1.02 & $\mathrm{~b}$ & 1.06 & $\mathrm{~d}$ & 2.43 & $\mathrm{~b}$ & 2.79 & $\mathrm{~b}$ \\
\hline 6 & 1.22 & $\mathrm{a}$ & 1.14 & $\mathrm{c}$ & 2.53 & $\mathrm{~b}$ & 3.19 & $\mathrm{a}$ & 46 & 1.24 & $\mathrm{a}$ & 1.28 & $\mathrm{~b}$ & 2.99 & $\mathrm{a}$ & 3.09 & $\mathrm{a}$ \\
\hline 7 & 1.01 & $\mathrm{~b}$ & 1.12 & $\mathrm{c}$ & 2.69 & $\mathrm{~b}$ & 2.85 & $\mathrm{a}$ & 47 & 0.99 & $\mathrm{~b}$ & 1.06 & $\mathrm{~d}$ & 2.51 & $\mathrm{~b}$ & 2.76 & $\mathrm{~b}$ \\
\hline 8 & 1.29 & $\mathrm{a}$ & 1.30 & $\mathrm{~b}$ & 2.48 & $\mathrm{~b}$ & 2.93 & $\mathrm{a}$ & 48 & 1.04 & $\mathrm{~b}$ & 0.97 & $\mathrm{~d}$ & 2.56 & $\mathrm{~b}$ & 3.01 & $\mathrm{a}$ \\
\hline 9 & 1.12 & $\mathrm{a}$ & 1.13 & c & 2.50 & $\mathrm{~b}$ & 2.98 & $\mathrm{a}$ & 49 & 1.13 & $\mathrm{a}$ & 1.11 & c & 2.56 & $\mathrm{~b}$ & 2.77 & $\mathrm{~b}$ \\
\hline 10 & 1.07 & $\mathrm{~b}$ & 1.03 & $\mathrm{~d}$ & 2.48 & $\mathrm{~b}$ & 2.71 & $\mathrm{~b}$ & 50 & 0.91 & $\mathrm{~b}$ & 1.02 & $\mathrm{~d}$ & 2.63 & $\mathrm{~b}$ & 3.02 & $\mathrm{a}$ \\
\hline 11 & 0.80 & $\mathrm{~b}$ & 0.87 & $\mathrm{e}$ & 2.27 & $\mathrm{~b}$ & 2.44 & $\mathrm{~b}$ & 51 & 1.18 & $\mathrm{a}$ & 1.16 & $\mathrm{c}$ & 3.12 & $\mathrm{a}$ & 3.62 & $\mathrm{a}$ \\
\hline 12 & 1.00 & $\mathrm{~b}$ & 1.04 & $\mathrm{~d}$ & 2.16 & $\mathrm{~b}$ & 2.74 & $\mathrm{~b}$ & 52 & 1.10 & $\mathrm{a}$ & 1.19 & $\mathrm{c}$ & 3.06 & $\mathrm{a}$ & 3.36 & $\mathrm{a}$ \\
\hline 13 & 1.43 & $\mathrm{a}$ & 1.45 & $\mathrm{a}$ & 2.75 & $\mathrm{a}$ & 3.22 & $\mathrm{a}$ & 53 & 1.21 & $\mathrm{a}$ & 1.22 & c & 2.97 & $\mathrm{a}$ & 3.30 & $\mathrm{a}$ \\
\hline 14 & 1.07 & $\mathrm{~b}$ & 1.22 & $\mathrm{c}$ & 2.38 & $\mathrm{~b}$ & 2.96 & $\mathrm{a}$ & 54 & 0.96 & $\mathrm{~b}$ & 0.85 & $\mathrm{e}$ & 2.45 & $\mathrm{~b}$ & 2.98 & $\mathrm{a}$ \\
\hline 15 & 1.06 & $\mathrm{~b}$ & 1.09 & c & 2.36 & $\mathrm{~b}$ & 2.69 & $\mathrm{~b}$ & 55 & 1.07 & $\mathrm{~b}$ & 1.03 & $\mathrm{~d}$ & 2.79 & $\mathrm{a}$ & 2.75 & $\mathrm{~b}$ \\
\hline 16 & 1.08 & $\mathrm{~b}$ & 1.27 & $\mathrm{~b}$ & 2.82 & $\mathrm{a}$ & 2.85 & $\mathrm{a}$ & 56 & 1.14 & $\mathrm{a}$ & 1.23 & $\mathrm{~b}$ & 2.15 & $\mathrm{~b}$ & 2.79 & $\mathrm{~b}$ \\
\hline 17 & 0.91 & $\mathrm{~b}$ & 1.20 & $\mathrm{c}$ & 3.10 & $\mathrm{a}$ & 2.93 & $\mathrm{a}$ & 57 & 0.94 & $\mathrm{~b}$ & 1.03 & $\mathrm{~d}$ & 2.47 & $\mathrm{~b}$ & 2.57 & $\mathrm{~b}$ \\
\hline 18 & 1.16 & $\mathrm{a}$ & 1.19 & $\mathrm{c}$ & 2.63 & $\mathrm{~b}$ & 3.10 & $\mathrm{a}$ & 58 & 1.09 & $\mathrm{~b}$ & 1.12 & $\mathrm{c}$ & 2.97 & $\mathrm{a}$ & 3.22 & $\mathrm{a}$ \\
\hline 19 & 1.19 & $\mathrm{a}$ & 1.15 & $\mathrm{c}$ & 1.59 & $\mathrm{~b}$ & 2.52 & $\mathrm{~b}$ & 59 & 0.88 & $\mathrm{~b}$ & 0.94 & $\mathrm{~d}$ & 3.19 & $\mathrm{a}$ & 3.61 & $\mathrm{a}$ \\
\hline 20 & 1.23 & $\mathrm{a}$ & 1.19 & c & 1.84 & $\mathrm{~b}$ & 2.62 & $\mathrm{~b}$ & 60 & 0.99 & $\mathrm{~b}$ & 1.07 & $\mathrm{~d}$ & 2.81 & $\mathrm{a}$ & 2.94 & $\mathrm{a}$ \\
\hline 21 & 1.07 & $\mathrm{~b}$ & 1.04 & $\mathrm{~d}$ & 2.08 & $\mathrm{~b}$ & 2.75 & $\mathrm{~b}$ & 61 & 1.11 & $\mathrm{a}$ & 1.11 & c & 2.85 & $\mathrm{a}$ & 3.29 & $\mathrm{a}$ \\
\hline 22 & 1.24 & $\mathrm{a}$ & 1.17 & $\mathrm{c}$ & 2.43 & $\mathrm{~b}$ & 2.80 & $\mathrm{~b}$ & 62 & 0.96 & $\mathrm{~b}$ & 1.02 & $\mathrm{~d}$ & 2.99 & $\mathrm{a}$ & 2.92 & $\mathrm{a}$ \\
\hline 23 & 1.05 & $\mathrm{~b}$ & 1.04 & $\mathrm{~d}$ & 2.59 & $\mathrm{~b}$ & 2.74 & $\mathrm{~b}$ & 63 & 1.03 & $\mathrm{~b}$ & 0.98 & $\mathrm{~d}$ & 2.68 & $\mathrm{~b}$ & 2.48 & $\mathrm{~b}$ \\
\hline 24 & 1.45 & $\mathrm{a}$ & 1.43 & $\mathrm{a}$ & 2.44 & $\mathrm{~b}$ & 2.94 & $\mathrm{a}$ & 64 & 1.04 & $\mathrm{~b}$ & 1.02 & $\mathrm{~d}$ & 2.91 & $\mathrm{a}$ & 2.97 & $\mathrm{a}$ \\
\hline 25 & 1.40 & $\mathrm{a}$ & 1.30 & $\mathrm{~b}$ & 2.35 & $\mathrm{~b}$ & 2.59 & $\mathrm{~b}$ & 65 & 0.85 & $\mathrm{~b}$ & 0.87 & $\mathrm{e}$ & 2.13 & $\mathrm{~b}$ & 2.30 & $\mathrm{c}$ \\
\hline 26 & 1.02 & $\mathrm{~b}$ & 1.03 & $\mathrm{~d}$ & 2.24 & $\mathrm{~b}$ & 2.44 & $\mathrm{~b}$ & 66 & 1.09 & $\mathrm{~b}$ & 1.10 & c & 3.38 & $\mathrm{a}$ & 2.76 & $\mathrm{~b}$ \\
\hline 27 & 1.05 & $\mathrm{~b}$ & 1.13 & $\mathrm{c}$ & 2.34 & $\mathrm{~b}$ & 2.57 & $\mathrm{~b}$ & 67 & 1.07 & $\mathrm{~b}$ & 1.01 & d & 2.29 & $\mathrm{~b}$ & 2.58 & $\mathrm{~b}$ \\
\hline 28 & 0.70 & $\mathrm{~b}$ & 0.67 & $\mathrm{f}$ & 2.11 & $\mathrm{~b}$ & 1.86 & $\mathrm{c}$ & 68 & 1.17 & $\mathrm{a}$ & 1.25 & b & 3.16 & $\mathrm{a}$ & 3.19 & $\mathrm{a}$ \\
\hline 29 & 0.83 & $\mathrm{~b}$ & 0.85 & $\mathrm{e}$ & 2.33 & $\mathrm{~b}$ & 2.06 & $\mathrm{c}$ & 69 & 0.71 & $\mathrm{~b}$ & 0.75 & $\mathrm{f}$ & 2.30 & $\mathrm{~b}$ & 2.21 & $\mathrm{c}$ \\
\hline 30 & 1.18 & $\mathrm{a}$ & 0.99 & $\mathrm{~d}$ & 2.18 & $\mathrm{~b}$ & 2.16 & $\mathrm{c}$ & 70 & 0.85 & $\mathrm{~b}$ & 0.88 & $\mathrm{e}$ & 2.88 & $\mathrm{a}$ & 2.53 & $\mathrm{~b}$ \\
\hline 31 & 1.25 & $\mathrm{a}$ & 1.30 & $\mathrm{~b}$ & 2.49 & $\mathrm{~b}$ & 1.98 & $\mathrm{c}$ & 71 & 0.99 & $\mathrm{~b}$ & 0.97 & $\mathrm{~d}$ & 2.63 & $\mathrm{~b}$ & 2.61 & $\mathrm{~b}$ \\
\hline 32 & 1.04 & $\mathrm{~b}$ & 0.85 & $\mathrm{e}$ & 2.64 & $\mathrm{~b}$ & 2.53 & $\mathrm{~b}$ & 72 & 0.80 & $\mathrm{~b}$ & 1.08 & $\mathrm{c}$ & 2.81 & $\mathrm{a}$ & 2.54 & $\mathrm{~b}$ \\
\hline 33 & 0.95 & $\mathrm{~b}$ & 1.00 & $\mathrm{~d}$ & 2.12 & $\mathrm{~b}$ & 2.52 & $\mathrm{~b}$ & 73 & 1.08 & $\mathrm{~b}$ & 1.12 & $\mathrm{c}$ & 3.13 & $\mathrm{a}$ & 2.91 & $\mathrm{a}$ \\
\hline 34 & 1.01 & $\mathrm{~b}$ & 1.14 & $\mathrm{c}$ & 3.13 & $\mathrm{a}$ & 2.87 & $\mathrm{a}$ & 74 & 1.06 & $\mathrm{~b}$ & 1.08 & $\mathrm{c}$ & 2.99 & $\mathrm{a}$ & 2.97 & $\mathrm{a}$ \\
\hline 35 & 0.89 & $\mathrm{~b}$ & 0.94 & $\mathrm{~d}$ & 2.49 & $\mathrm{~b}$ & 2.87 & $\mathrm{a}$ & 75 & 0.95 & $\mathrm{~b}$ & 1.09 & c & 2.67 & $\mathrm{~b}$ & 2.61 & $\mathrm{~b}$ \\
\hline 36 & 0.99 & $\mathrm{~b}$ & 1.14 & c & 2.64 & $\mathrm{~b}$ & 3.04 & $\mathrm{a}$ & 76 & 0.97 & $\mathrm{~b}$ & 1.05 & $\mathrm{~d}$ & 2.74 & $\mathrm{a}$ & 2.68 & $\mathrm{~b}$ \\
\hline 37 & 1.11 & $\mathrm{a}$ & 1.24 & $\mathrm{~b}$ & 3.05 & $\mathrm{a}$ & 3.23 & $\mathrm{a}$ & 77 & 1.21 & $\mathrm{a}$ & 1.32 & $\mathrm{~b}$ & 2.98 & $\mathrm{a}$ & 2.77 & $\mathrm{~b}$ \\
\hline 38. & 1.03 & $\mathrm{~b}$ & 0.97 & $\mathrm{~d}$ & 3.14 & $\mathrm{a}$ & 3.27 & $\mathrm{a}$ & 78 & 0.96 & $\mathrm{~b}$ & 0.99 & $\mathrm{~d}$ & 3.02 & $\mathrm{a}$ & 2.55 & $\mathrm{~b}$ \\
\hline 39 & 0.95 & $\mathrm{~b}$ & 0.90 & $\mathrm{e}$ & 2.68 & $\mathrm{~b}$ & 2.70 & $\mathrm{~b}$ & 79 & 0.87 & $\mathrm{~b}$ & 0.91 & $\mathrm{e}$ & 2.90 & $\mathrm{a}$ & 2.85 & $\mathrm{a}$ \\
\hline 40 & 1.05 & b & 1.11 & c & 3.20 & a & 3.22 & a & 80 & 0.99 & b & 1.19 & c & 3.35 & $\mathrm{a}$ & 3.08 & $\mathrm{a}$ \\
\hline Mean Dry & 1.06 & & & & 2.64 & & & & Mean Rainy & 1.09 & & & & 2 & 2. & & \\
\hline
\end{tabular}

Note. ${ }^{1}$ Values followed by the same letter within columns do not differ by Scott-Knott's test at 5\% probability. 


\subsection{Multivariate Analysis}

When a set of traits represents qualitatively different variables and there is no correlation between them, univariate analysis is the most appropriate procedure to apply; however, when a dataset displays variables that are correlated, multinormality should be assumed, and a multivariate analysis of variance should be performed (Freitas et al., 2000).

For forage plants, a multivariate analysis is recommended, since traits usually display an appreciable level of correlation, being measured in the same tussock (Freitas et al., 2000).

When this analysis was performed on our data, the variance accumulated by the first two canonical variables was $66.8 \%$ of the total variance (Table 5), which made it possible to adopt this technique in the study of scattering of genotypes on a 2-dimensional graph. Evaluating the phenotypic divergence among 99 genotypes of elephant grass in Coronel Pacheco/MG (Brazil), Shimoya et al. (2002) observed that the first 2 canonical variables explained only $50.0 \%$ of the total variance. However, Daher et al. (1997) obtained a total of $80.7 \%$ of the variation in the first 2 canonical variables using 17 genotypes of elephant grass in Campos dos Goytacazes/RJ, and inferred that there was genetic divergence among the genotypes.

Table 5. Estimates of variances (eigenvalues, $\lambda_{\mathrm{j}}$ ) associated with the canonical variables, and respective weight coefficients (eigenvectors) of 8 variables assessed in 80 genotypes of elephant grass in Campos dos Goytacazes/RJ, Brazil

\begin{tabular}{llllllllll}
\hline$\lambda_{\mathrm{j}}$ & \multirow{2}{*}{ Accumulated variance $(\%)$} & \multicolumn{9}{c}{ Variables } \\
\cline { 3 - 10 } & & $(1)$ & $(2)$ & $(3)$ & $(4)$ & $(5)$ & $(6)$ & $(7)$ & $(8)$ \\
\hline 7.358 & 45.488 & -0.141 & -0.039 & -0.088 & 0.072 & 0.373 & 0.733 & 0.456 & 0.284 \\
3.457 & 66.860 & 0.007 & 0.006 & 0.152 & -0.221 & -0.445 & -0.313 & 0.644 & 0.466 \\
1.752 & 77.693 & -0.575 & -0.007 & 0.533 & 0.148 & -0.010 & 0.014 & -0.397 & 0.454 \\
1.263 & 85.504 & 0.176 & 0.079 & 0.620 & 0.452 & -0.260 & 0.215 & 0.251 & -0.443 \\
0.946 & 91.349 & 0.404 & -0.371 & 0.004 & 0.588 & 0.348 & -0.329 & 0.026 & 0.351 \\
0.604 & 95.085 & -0.380 & 0.510 & -0.444 & 0.576 & -0.135 & -0.167 & 0.139 & -0.017 \\
0.429 & 97.734 & 0.489 & 0.750 & 0.173 & -0.099 & 0.159 & 0.069 & -0.171 & 0.313 \\
0.366 & 100.000 & 0.265 & -0.178 & -0.272 & 0.179 & -0.656 & 0.419 & -0.327 & 0.280 \\
\hline
\end{tabular}

Note. 1 = NT in Dry, 2 = NT in Rainy; 3 = HGT in Dry, $4=$ HGT in Rainy, in m; 5 = SD in Dry, 6 = SD in Rainy, in $\mathrm{mm} ; 7=\mathrm{LBW}$ in Dry; and $8=\mathrm{LBW}$ in Rainy, in $\mathrm{cm}$.

In the clustering analysis of the 80 genotypes, 15 Groups were formed with Group 1 containing 39 genotypes, Group 2 having 14 Genotypes, Group 6 having 7 genotypes and 7 of the remaining groups having only a single genotype (Table 6; Figure 1). The genotypes that comprised the elite group based on dry matter yields, described previously, were contained in Groups 1,2, 3, 4 and 10.

Table 6. Clustering of 80 genotypes of elephant grass into groups by Tocher's optimization method based on generalized Mahalanobis distance $\left(D^{2}\right)$ (Campos dos Goytacazes/RJ, Brazil, 2012/2013)

\begin{tabular}{ll}
\hline Group & Genotypes \\
\hline 1 & 101223474955221501425044091460757641355771616258647874732726676306792145563366 \\
2 & 5821853374643521677513640 \\
3 & 192025 \\
4 & 1324 \\
5 & 3859 \\
6 & 11296570543239 \\
7 & 43473 \\
8 & 1772 \\
9 & 80 \\
10 & 68 \\
11 & 31 \\
12 & 48 \\
13 & 30 \\
14 & 69 \\
15 & 28 \\
\hline
\end{tabular}




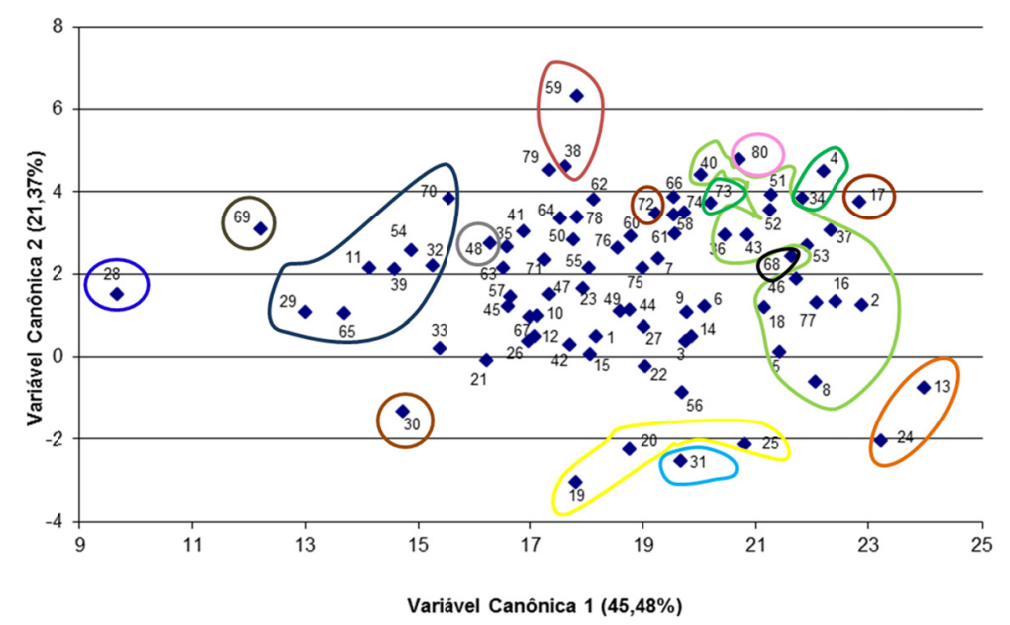

Figure 1. Scattering diagram of 80 genotypes of elephant grass, obtained by analysis of canonical variables. Legend: G1 = no color; G2 = light green; G3 = yellow; G4 = orange; G5 = dark red; G6 = dark blue; G7 = dark green; G8 = dark brown; G9 = pink; G10 = black; G11 = light blue; G12 = light gray; G13= light brown; G14= dark gray; G15 = royal blue

\section{Conclusion}

The genotypes that comprised the elite group were Elefante da Colômbia, Taiwan A-25, Albano, Hibrido Gigante da Colômbia, Elefante de Pinda, Taiwan A-121, P241 Piracicaba, Guaçu/I.Z.2, CPAC, EMPASC 309, EMPASC 307, Australiano, and Pasto Panamá. Future evaluations of the bromatological quality of these genotypes should be carried out.

Clustering analysis provided direction for crossings involving five groups; stem diameter (rainy season) and leaf blade width (dry season) were the most important variables to explain the scattering of genotypes.

\section{Acknowledgments}

The authors thank Fundação Carlos Chagas Filho de Amparo à Pesquisa do Estado do Rio de Janeiro (FAPERJ) for financing this research.

\section{References}

Azevedo, A. L. S., Costa, P. P., Machado, J. C., Machado, M. A., Pereira, A. V., \& Silva, L. F. J. (2012). Cross species amplification of Pennisetum glaucum microsatellite markers in Pennisetum purpureum and genetic diversity of Napier grass accessions. Crop Science, 52, 1776-1785. https://doi.org/10.2135/cropsci2011. 09.0480

Cavalcante, M., \& Lira, M. A. (2010). Variabilidade genética em Pennisetum purpureum Schumacher. Revista Caatinga, 23, 153-163.

Cunha, M. V., Lira, M. A., Santos, M. V., Dubeux Júnior, J. C. B., Mello, A. C. L., \& Freitas, E. V. (2013). Adaptabilidade e estabilidade da produção de forragem por meio de diferentes metodologias na seleção de clones de Pennisetum spp. Revista Brasileira Ciências Agrárias, 8, 681-686. https://doi.org/10.5039/ agraria.v8i4a3280

Cunha, R. C. V. (2012). Análise de estabilidade da produção forrageira de genótipos de capim-elefante avaliados em Campos dos Goytacazes-RJ (Master's thesis, UENF, Campos dos Goytacazes).

Cruz, C.D. (2013). GENES: A software package for analysis in experimental statistics and quantitative genetics. Acta Scientiarum. Agronomy, 35, 271-276. http://dx.doi.org/10.4025/actasciagron.v35i3.21251

Daher, R. F, Moraes, C. F., Cruz, C. D., Pereira, A. V., \& Xavier, D. F. (1997). Seleção de caracteres morfológicos discriminantes em capim-elefante (Pennisetum purpureum Schum.). Revista Brasileira de Zootecnia, 26, 265-270.

Daher, R. F., Pereira, A. V., Pereira, M. G., Lédo, F. J. S., Amaral Junior, A. T., Rocabado, J. M. A., ... Tardin, F. D. (2004). Análise de trilha de caracteres forrageiros do capim-elefante (Pennisetum purpureum Schum.). Ciencia Rural, 34, 1531-1535. https://doi.org/10.1590/S0103-84782004000500032 
Embrapa (Empresa Brasileira de Pesquisa Agropecuária). (2006). Sistema brasileiro de classificação de solos (p. 412). Rio de Janeiro: EMBRAPA.

Ferreira, A. C. H., Neiva, J. N. M., Rodriguez, N. M., Lopes, F. C. F., \& Lôbo, R. N. B. (2010). Consumo e digestibilidade de silagens de capim-elefante com diferentes níveis de subproduto da agroindústria da acerola. Revista Ciência Agronômica, 41, 693-701. https://doi.org/10.1590/S1806-66902010000400025

Freitas, N. S. A., Falcão, T. M. M. A., Burity, H. A., Tabosa J. N., \& Silva, M. V. (2000). Caracterização e diversidade genética do capim-elefante seus híbridos com milheto mediante padrões isoenzimáticos. Pesquisa Agropecuária Brasileira, 35, 1125-1133. https://doi.org/10.1590/S0100-204X2000000600008

Garcia, C. S., Fernandes, A. M., Fontes, C. A. A., Vieira, R. A. M.; Sant'Ana, N. F., \& Pimentel, V. A. (2011). Desempenho de novilhos mantidos em pastagens de capim-elefante e capim-mombaça. Revista Brasileira de Zootecnia, 40, 403-410. https://doi.org/10.1590/S1516-35982011000200023

Leão, F. F., Cancellier, L. L., Pereira, A. V., Ledo, F. J. S., \& Afférri, F. S. (2012). Produção forrageira e composição bromatológica de combinações genômicas de capim-elefante e milheto. Revita Ciencia Agronomica, 43, 368-375. https://doi.org/10.1590/S1806-66902012000200021

Lima, R. S. N., Daher, R. F., Goncalves, L. S. A., Rossi, D. A., Amaral Júnior, A. T., Pereira, M. G., \& Lédo, F. J. (2011). RAPD and ISSR markers in the evaluation of genetic divergence among accessions of elephant grass. Genetics and Molecular Research, 10, 1304-13.

Lista, F. N. (2008). Avaliação de cultivares de capim-elefante (Pennisetum purpureum Schum.) para ruminantes na região Norte Fluminense (Doctoral thesis, UENF, Campos dos Goytacazes).

Rezende, C. P., Pereira, J. M., Cardoso, P. J., Muniz, J. Á., Borges, A. M. F., Andrade, I. F., \& Evangelista, A. R. (2008). Dinâmica de perfilhamento e fluxo de biomassa em capim-cameroon sob lotação rotativa. Revista Brasileira de Zootecnia, 37, 1750-1757. https://doi.org/10.1590/S1516-35982008001000006

Santos, E. A., Silva, D. S., \& Queiroz Filho, J. L. (2001). Composição química do capim-elefante cv. Roxo cortado em diferentes alturas. Revista Brasileira de Zootecnia, 30, 18-23. https://doi.org/10.1590/S151635982001000100004

Silva, S. H. B., Santos, M. V. F., Lira, M. A., Dubeux Junior, J. C. B., Freitas, E. V., \& Ferreira, R. L. C. (2009). Uso de descritores morfológicos e herdabilidade de caracteres em clones de capim-elefante de porte baixo. Revista Brasileira de Zootecnia, 38, 1516-3598. https://doi.org/10.1590/S1516-35982009000800008

Silva, V. Q. R. (2011). Dialelo parcial em capim-elefante: Capacidade combinatória em caracteres morfoagronômicos e bromatológicos em Campos dos Goytacazes, RJ (Doctoral thesis, UENF, Campos dos Goytacazes).

Shimoya, A., Cruz, C. D., Ferreira, R. P., Pereira, A. V., \& Carneiro, P. C. S. (2002). Divergência genética entre acessos de um banco de germoplasma. Pesquisa Agropecuária Brasileira, 37, 971-980. https://doi.org/ 10.1590/S0100-204X2002000700011

Souza Sobrinho, F., Pereira, A. V., Ledo, F. J. S., Botrel, M. A., Oliveira, J. S., \& Xavier, D. F. (2005). Avaliação agronômica de híbridos interespecíficos entre capim-elefante e milheto. Pesquisa Agropecuária Brasileira, 40, 873-880. https://doi.org/10.1590/S0100-204X2005000900006

Vasconcelos, E. S., Cruz, C. D., Bhering, L. L., \& Resende Júnior, M. F. R. (2011). Método alternativo para análise de agrupamento. Pesquisa Agropecuária Brasileira, 42, 1421-1428. https://doi.org/10.1590/S0100204X2007001000008

Zhang, X., Gu, H., Ding, C., Zhong, X., Zhang, J., \& Xu, Nengxiang. (2010). Path coefficient and cluster analyses of yield and morphological traits in Pennisetum purpureum. Tropical Grassland, 44, 95-102.

\section{Copyrights}

Copyright for this article is retained by the author(s), with first publication rights granted to the journal.

This is an open-access article distributed under the terms and conditions of the Creative Commons Attribution license (http://creativecommons.org/licenses/by/4.0/). 